\title{
A NOTE ON OPTIMAL REINSURANCE
}

\author{
Gunnar Benktander \\ Zurich
}

I In the past the subject of optimal reinsurance has been dealt with by various authors such as Borch (I), Kahn (2) and Verbeek (3). Borch tries to look at the problem from the point of view of a company which acts as both Insurer and Reinsurer whereas the viewpoint of Kahn and Verbeek is that of a Ceding Company.

2 Let us study the simplest possible market situation of one Insurance Company $C$ and one Reinsurer $R$. $C$ writes a portfolio $P$ and seeks reinsurance protection at $R$.

In the present note I will start with the viewpoint of optimizing simultaneously the situation of $C$ and $R$.

3 For both $C$ and $R$ the variance is used as a measure of risk. The variance of $P$ is $V . C$ makes an effort to reduce $V$ to a level $V_{C}$ by way of a reinsurance treaty $T$. The corresponding variance of $R$ is $V_{R}$.

4 Out of the set of possible reinsurance treaty arrangements $\{T\}$ we assume that the parties are first of all interested in such arrangements for which

$$
V-V_{C}-V_{R} \gg 0 .
$$

In other words they will look for solutions which will lead to a substantial reduction of the variance.

It is possible to think of "nonsense" arrangements where no substantial reduction is achieved and in the extreme case even of arrangements for which

$$
V_{C}+V_{R}>V \text {. }
$$

In a further paper we might deal with such a case.

$5 C$ 's risk aversion is expressed by $a_{C}$ and $R$ 's by $a_{R} . a_{C}$ and $a_{R}$ have the dimension - $\mathrm{I}$. The maximum "price" in the form of a loading addition to the pure risk premium which $C$ is prepared to pay to $R$ is

$$
\frac{1}{2} a_{C}\left(V-V_{C}\right)
$$


and the minimum "price" which is acceptable for $R$ is

$$
\frac{1}{2} a_{R} \cdot V_{R} \text {. }
$$

The common mathematical definition of the risk aversion $a(K)$ as a function of the existing reserves $K$ is

$$
a(K)=-\frac{u^{\prime \prime}(K)}{u^{\prime}(K)}
$$

where $u$ is the utility of money.

Under rather general assumptions an addition to the pure risk premium based on utility theory is approximately

$$
\frac{1}{2} a . V \text {. }
$$

A strict proof of this would necessitate a separate paper.

Among all possible treaties $\{T\}$ we thus have to concentrate on those for which

$$
a_{C}\left(V-V_{C}\right)>a_{R} V_{R}
$$

In this simplified model we neglect the cost for negotiating and administering the reinsurance.

6 We assert that it is always possible to find a treaty $T_{1} \varepsilon\{T\}$ such that

$$
a_{C}\left(V-V_{C}\right)>a_{R} \cdot V_{R}
$$

Proof: This inequality can be written

$$
V-V_{C}-\frac{a_{R}}{a_{C}} \cdot V_{R}>0 .
$$

We can always choose $T_{1}$ in such a way that

$$
V-V_{C}-V_{R}>B>0 \text {. }
$$

a) Suppose $a_{R}<a_{C}$. This will be the normal case when the Reinsurer has stronger inner reserves than the Ceding Company.

We then have

$$
V-V_{C}-\frac{a_{R}}{a_{C}} \cdot V_{R}>V-V_{C}-V_{R}>B>0 .
$$


b) When a small Reinsurer deals with a big and strong Ceding Company we probably have $a_{R}>a_{C}$,

and

$$
V-V_{C}-\frac{a_{R}}{a_{C}} \cdot V_{R}>B+V_{R}-\frac{a_{R}}{a_{C}} \cdot V_{R}=B-\frac{a_{R}-a_{C}}{a_{C}} \cdot V_{R} .
$$

This is obviously positive if we limit the quantity of reinsurance in such a way that

$$
V_{R}<\frac{B \cdot a_{C}}{a_{R}-a_{C}} .
$$

The above means that there will always be an interest for the two parties to arrange a reinsurance treaty.

7 Suppose for a moment that $a_{C}=a_{R}=a$.

It then appears as natural to seek a treaty out of $\{T\}$ such that $V-V_{C}-V_{R}$ is a maximum.

To divide this variance reduction evenly between the parties seems to be fair.

We always have

$$
V-V_{C}-V_{R}=2 \rho_{C R} \cdot \sqrt{V_{C} \cdot V_{R}}
$$

The correlation in results between $C$ and $R, \rho_{C R}$, is obviously a maximum when $C$ cedes a quota share to $R$.

$\sqrt{V_{C} V_{R}}$ is maximum when the quota share is $50 \%$.

Through the reinsurance arrangement we have annihilated a variance equal to $0.50 \mathrm{~V}$ and divided the remaining variance between the two parties.

The maximum price is $\pi \max .=(a / 2) \cdot\left(V-V_{C}\right)=a \cdot 0.375 \cdot V$ and

the minimum price is $\pi \min .=(a / 2) \cdot V_{R} \quad=a \cdot 0.125 \cdot V$

A fair reinsurance "price" is $a .0 .25$. $V$ which is equal to double the minimum price of $R$ and two thirds of the maximum price of $C$.

8 Suppose that $a_{C} \neq a_{R}$.

$$
\begin{aligned}
& \pi \max .=\frac{1}{2} a_{C}\left(V-V_{C}\right) \\
& \pi \text { min. }=\frac{1}{2} a_{R} \cdot V_{R} .
\end{aligned}
$$


It makes sense to seek a reinsurance treaty arrangement which maximizes the difference $D=\pi \max$. $-\pi \min$.

The parties agree on a quota share arrangement which they have already found is optimal in the case of $a_{C}=a_{R}$.

$C$ retains a share $k$ and cedes $\mathrm{I}-k$ to $R$.

$$
\begin{aligned}
& D=\frac{1}{2} a_{C}\left(V-V_{C}\right)-\frac{1}{2} a_{R} V_{R}=\frac{1}{2} a_{C}\left(V-k^{2} V\right)-\frac{1}{2} a_{R}(\mathrm{I}-k)^{2} V= \\
& \quad=\frac{1}{2} V\left[a_{C}-a_{R}+2 k a_{R}-k^{2}\left(a_{C}+a_{R}\right)\right] \\
& \frac{\partial D}{\partial k}=\text { o gives } k=\frac{a_{R}}{a_{C}+a_{R}} ; \mathrm{I}-k=\frac{a_{C}}{a_{C}+a_{R}} ; \frac{k}{\mathrm{I}-k}=\frac{a_{R}}{a_{C}}
\end{aligned}
$$

Thus, not unexpectedly, the shares between Ceding Company and Reinsurer will be partitioned in inverse proportion to their respective risk aversion.

$$
D \max .=\frac{1}{2} V\left[a_{C}-a_{R}+\frac{2 a_{R}^{2}}{\left(a_{C}+a_{R}\right)}-\frac{a_{R}^{2}}{a_{C}+a_{R}}\right]=\frac{1}{2} V \cdot \frac{a_{C}^{2}}{\left(a_{C}+a_{R}\right)}
$$

The above value of $k$ leads to

$$
\pi \max .=\frac{1}{2} a_{C} \cdot V\left(\mathrm{I}-k^{2}\right)=\frac{1}{2} a_{C} V \cdot\left[\mathrm{I}-\left(\frac{a_{R}}{a_{C}+a_{R}}\right)^{2}\right]
$$

and

$$
\pi \min .=\frac{1}{2} a_{R} \cdot V(\mathrm{I}-k)^{2}=\frac{1}{2} a_{R} V \cdot\left(\frac{a_{C}}{a_{C}+a_{R}}\right)^{2}
$$

The parties will have to find a price between these two extremes. A "fair" price could be $\pi$ min. $+\frac{1}{2} D$ max.

9 When $V$ is used as risk measure it is obvious that quota share reinsurance has some good characteristics. This is so because of the fact that a substantial part of the variance disappears due to the high correlation between the results for $C$ and $R$. In practice $C$ is eager to limit his total expenditure for reinsurance, which excludes a quota share treaty. Let us therefore look into the case when the parties agree to base their relations on excess of loss reinsurance. $R$ covers the layer xs a certain retention $m>m_{0}$ up to a certain limit $M_{1}$ at a flat rate. The importance of the last condition might be illustrated in a future paper. Let us assume that losses up to an amount $m_{0}$ under all circumstances are covered by $C$ and in this context disregard the variations in results in that interval, whether 
their causes be purely stochastic or due to varying basic probabilities. Let us further assume that the number of claims in xs of $m_{0}$ is Poisson distributed with an expected value $n$.

The total variance in $x s$ of $m_{0}$ is $V$ in layer I i.e. $m_{0}-m$ it is $V_{\mathbf{I}}$ and in layer II i.e. $m-M_{1}$ it is $V_{\mathrm{II}}$.

Claims in $x s$ of $M_{1}$ either do not exist or are covered by means of protection arranged on a national basis and can thus be ignored in this context.

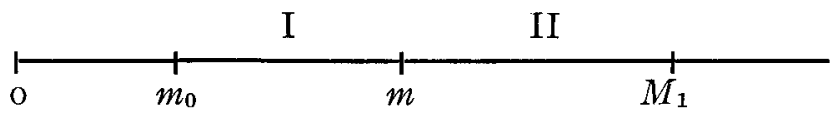

Io Let us study the case when the claims size distribution is Pareto with an $\alpha>2$.

Then we have putting $m_{0}=\mathrm{I}$

$$
\begin{array}{r}
V=\frac{2 n}{(\alpha-\mathrm{I})(\alpha-2)} \cdot\left[\mathrm{I}-M_{1}^{-(\alpha-2)}\left(\alpha-\mathrm{I}-\frac{\alpha-2}{M_{1}}\right)\right] \\
V_{\mathrm{I}}=\frac{2 n}{(\alpha-\mathrm{I})(\alpha-2)} \cdot\left[\mathrm{I}-m^{-(\alpha-2)}\left(\alpha-\mathrm{I}-\frac{\alpha-2}{m}\right)\right]
\end{array}
$$

and

$V_{\mathrm{II}}=\frac{2 n}{(\alpha-\mathrm{I})(\alpha-2)} \quad m^{-(\alpha-2)}-M_{1}^{-(\alpha-2)}\left(\alpha-\mathrm{I}-\frac{(\alpha-2) m}{M_{1}}\right)$

The total variance reduction then is

$$
\begin{aligned}
& V-V_{\mathrm{I}}-V_{\mathrm{II}}=\frac{2 n}{(\alpha-\mathrm{I})(\alpha-2)} \cdot\left[m^{-(\alpha-2)}(\alpha-2)\left(\mathrm{I}-\frac{\mathrm{I}}{m}\right)-\right. \\
& \left.-M_{1}^{-(\alpha-2)} \cdot \frac{m-\mathrm{I}}{M_{1}}(\alpha-2)\right]=\frac{2 n}{\alpha-\mathrm{I}}(m-\mathrm{I})\left[\frac{\mathrm{I}}{m^{\alpha-1}}-\frac{\mathrm{I}}{M_{1}^{\alpha-1}}\right] .
\end{aligned}
$$

When $a_{C}=a_{R}$ it is natural to seek a value of $m$ such that the total variance reduction is a maximum. Also when $a_{C} \neq a_{R}$ this could make sense, at least as long as the size order of $a_{C}$ and $a_{R}$ is roughly same. 
II This leads us to differentiate $V-V_{\mathrm{I}}-V_{\text {II }}$ with regard to $m$

$$
\begin{aligned}
\frac{\partial}{\partial m} & =\frac{2 n}{\alpha-\mathrm{I}}\left[\frac{\mathrm{I}}{m^{\alpha-1}}-\frac{\mathrm{I}}{M_{1}^{\alpha-1}}-\frac{(m-\mathrm{I})(\alpha-\mathrm{I})}{m^{\alpha}}\right]= \\
& =\frac{2 n}{\alpha-\mathbf{I}}\left[\frac{\alpha-\mathrm{I}}{m^{\alpha}}-\frac{\alpha-2}{m^{\alpha-1}} \frac{\mathrm{I}}{M_{1}^{\alpha-1}}\right]
\end{aligned}
$$

If $M_{1}=\infty \quad \frac{\partial}{\partial m}=$ o gives $m=\frac{\alpha-\mathrm{I}}{\alpha-2}$

If $M_{1}<\infty \frac{\partial}{\partial m}=0$ gives $(\alpha-\mathrm{I})-m(\alpha-2)-\frac{m_{1}}{M_{1}^{\alpha-1}}=0$

$$
\begin{aligned}
m & =\frac{\alpha-\mathrm{I}}{\alpha-2}\left(\mathrm{I}-\frac{\mathrm{I}}{\alpha-\mathrm{I}} \cdot \frac{m^{\alpha}}{M_{1}^{\alpha-1}}\right) \\
& >\frac{\alpha-\mathrm{I}}{\alpha-2} \cdot\left[\mathrm{I}-\frac{\mathrm{I}}{\alpha-\mathrm{I}} \frac{\left(\frac{\alpha-\mathrm{I}}{\alpha-2}\right)^{\alpha}}{M_{1}^{\alpha-1}}\right] .
\end{aligned}
$$

In this case we thus have

$$
\frac{\alpha-\mathrm{I}}{\alpha-2}>m>\frac{\alpha-\mathrm{I}}{\alpha-2}\left[\mathrm{I}-\frac{\mathrm{I}}{\alpha-\mathrm{I}} \frac{\left(\frac{\alpha-\mathrm{I}}{\alpha-2}\right)^{\alpha}}{M_{1}^{\alpha-1}}\right] .
$$

If $\alpha=3$ and $M_{1}=$ Io this gives

$$
2>m>2 \cdot 0.96 \text {. }
$$

We have thus found that $V_{C}+V_{R}$ has a minimum for

$$
m \leq \frac{\alpha-\mathrm{I}}{\alpha-2} \text {. }
$$

If $M_{1}<\infty$ is not small, however, we use in practice $m=\frac{\alpha-\mathrm{I}}{\alpha-2}$ instead of the exact value.

The total risk reduction achieved by way of reinsurance, when related to the risk without reinsurance $V$, is equal to

$$
\frac{V-V_{\mathrm{I}}-V_{\mathrm{II}}}{V}=\frac{(\alpha-2)(m-\mathrm{I})}{m^{\alpha-1}} \cdot \frac{M_{1}^{\alpha-1}-m^{\alpha-1}}{M_{1}^{\alpha-1}-(\alpha-\mathrm{I}) M_{1}+\alpha-2} .
$$


For $M_{1}=\infty$ we obviously have

$$
\frac{V-V_{\mathrm{I}}-V_{\mathrm{II}}}{V}=\frac{(\alpha-2)(m-\mathrm{I})}{m^{\alpha-1}} .
$$

Inserting $m=\frac{\alpha-I}{\alpha-2}$ we obtain

$$
\frac{V-V_{\mathrm{I}}-V_{\mathrm{II}}}{V}=\left(\frac{\alpha-2}{\alpha-\mathrm{I}}\right)^{\alpha-1} \text {. }
$$

I2 $C$ 's risk reduction when $R$ covers Layer II is

$$
\begin{aligned}
V-V_{\mathbf{I}}=\frac{2 n}{(\alpha-\mathrm{I})(\alpha-2)}\left[m^{-\alpha+2}\left(\alpha-\mathrm{I}-\frac{\alpha-2}{m}\right)-\right. & \\
& \left.\quad-M_{1}^{-\alpha+2}\left(\alpha-\mathrm{I}-\frac{\alpha-2}{M_{1}}\right)\right] .
\end{aligned}
$$

If we put $M_{1}=\infty$ and insert $m=\frac{\alpha-\mathrm{I}}{\alpha-2}$ we obtain after some manipulation

$$
V-V_{\mathrm{I}}=2 n \cdot \frac{(\alpha-2)^{\alpha-8}}{(\alpha-\mathrm{I})^{\alpha}}(2 \alpha-3)
$$

The corresponding value of $V$ is $V=\frac{2 n}{(\alpha-\mathrm{I})(\alpha-2)}$

By way of reinsurance $C$ thus achieves a relative risk reduction

$$
\frac{V-V_{\mathrm{I}}}{V}=\frac{(\alpha-2)^{\alpha-2}}{(\alpha-\mathrm{I})^{\alpha-1}} \cdot(2 \alpha-3)=\left(\frac{\alpha-2}{\alpha-\mathrm{I}}\right)^{\alpha-2}\left(\mathrm{I}+\frac{\alpha-2}{\alpha-\mathrm{I}}\right) .
$$

The corresponding value of $R$ 's risk relative to $V$ is

$$
\frac{V \text { II }}{V}=\left(\frac{\alpha-2}{\alpha-1}\right)^{\alpha-2} \text {. }
$$

I3 The results of II and I2 above can be illustrated by the tables below for various values of $\alpha$ when $M_{1}=\infty$ : 
C's Relative risk reduction

retention

$R$ 's relative risk

$\begin{array}{lcccc}\alpha & \frac{\alpha-I}{\alpha-2} & \text { Total } & \text { For } C & \\ 2.25 & 5 & 0.14 & 0.80 & 0.67 \\ 2.5 & 3 & 0.19 & 0.77 & 0.58 \\ 3 & 2 & 0.25 & 0.75 & 0.50 \\ 4 & \text { r.5 } & 0.30 & 0.74 & 0.44 \\ 5 & \text { I.33 } & 0.32 & 0.74 & 0.42\end{array}$

Based on the above it appears sensible for the Reinsurer to ask the Ceding Company to run a higher retention the more dangerous and skew the claims distribution is (small values of $\alpha$ ).

The more dangerous the claims distribution is, the more efficient is the excess of loss reinsurance from $C$ 's point of view. $R$, however, has to take over a high amount of risk-in spite of the fact that $C$ has to hold a comparatively large amount for its own account. The total risk reduction, however, is not impressive which leads us to question $X L$ protection for dangerous portfolios and we ask whether a proportional arrangement would not be more adequate.

I4 The above results refer to the Pareto distribution. Let us make the corresponding calculations for a distribution which converges more quickly than Pareto.

We thus investigate the model

$$
\begin{aligned}
I-F(x)=H(x)=\text { const. } x^{-(1-b)} & e^{-(a / b) x^{b}} \\
& \text { for } 0<b<I
\end{aligned}
$$

which has been used by us to describe the claims size distribution in Motor insurance in excess of Swiss Francs roo.000, which amount is used as a unit.

For this distribution the expected value of the excess loss cost for the unlimited layer $x s x$ is

$$
E(x)=\text { const. } \cdot e^{-(a / b) x^{b}}
$$

and the average excess claim is

$$
m(x)=\frac{x^{1-b}}{a} .
$$


When $b=\mathrm{I}$ we obtain the exponential distribution as a borderline case, whereas $b=0$ leads to the Pareto distribution with $\alpha=a+$ I. (4)

I5 Let us limit ourselves to the special case $b=0.5$. Then the variance for the layer $\infty x s x$ is

$$
V(x)=2 n \int_{x} E(y) d y=E(x) \frac{n}{a^{2}}(\mathrm{I}+2 a \sqrt{x}) .
$$

With the same notation as in 9 above, and putting $m_{0}=\mathrm{I}$ we get

$$
\begin{aligned}
V & =\frac{n}{a^{2}}(\mathrm{I}+2 a) \cdot E(\mathrm{I}) \\
V_{\mathrm{I}} & =\frac{n}{a^{2}}\{-E(m)(\mathrm{I}+2 a \sqrt{m})+E(\mathrm{I})(\mathrm{I}+2 a)\}-2(m-\mathrm{I}) E(m) \cdot n \\
& =n\left\{(\mathrm{I}+2 a) \frac{\mathrm{I}}{a^{2}} E(\mathrm{I})-E(m)\left(2(m-\mathrm{I})+\frac{2 \sqrt{m}}{a}+\frac{\mathrm{I}}{a^{2}}\right)\right\}
\end{aligned}
$$

and

$V_{\mathrm{II}}=\frac{n}{a^{2}}(\mathrm{I}+2 a \sqrt{m}) \cdot E(m)$.

We have thus shown that the total variance reduction due to reinsurance is

$$
\begin{aligned}
V-V_{\mathbf{I}}-V_{\mathbf{I I}} & =n E(m)\left[\left(2(m-\mathbf{I})+\frac{2 \sqrt{m}}{a}+\frac{\mathrm{I}}{a^{2}}-\frac{\mathrm{I}}{a^{2}}-\frac{2 \sqrt{m}}{a}\right)\right]= \\
& =2 n(m-\mathbf{I}) E(m)=2 n(m-\mathbf{I}) e^{-2 a V \bar{m}} .
\end{aligned}
$$

I6 Differentiating this expression with regard to $m$ gives

$$
\frac{\partial}{\partial m}\left(V-V_{\mathrm{I}}-V_{\mathrm{II}}\right)=2 n E(m)\left\{\mathrm{I}-\frac{a}{\sqrt{m}}(m-I)\right\} .
$$

When $\frac{\partial}{\partial m}=$ o we get

$$
m=\frac{\mathrm{I}}{2 a^{2}}\left(\mathrm{I}+\sqrt{\mathrm{I}+4 a^{2}}\right)+\mathrm{I} .
$$

For $a=0,5: m=5,83$. 
Relative to the variance without reinsurance $V$ we get the total risk reduction

$$
\frac{V-V_{\mathrm{I}}-V_{\mathrm{II}}}{V}=\frac{2 a^{2}(m-\mathrm{I})}{\mathrm{I}+2 a} \frac{E(m)}{E(\mathrm{I})}=\frac{2 a^{2}(m-\mathrm{I})}{\mathrm{I}+2 a} e^{-2 a(\sqrt{m}-1)}
$$

C's risk reduction is

$$
\frac{V-V_{\mathrm{I}}}{V}=\frac{2(m-\mathrm{I}) a^{2}+2 \sqrt{m} a+\mathrm{I}}{\mathrm{I}+2 a} e^{-2 a\left(v_{\bar{m}}-1\right)}
$$

and $R$ 's risk is

$$
\frac{V_{\text {II }}}{V}=\frac{E(m)}{E(\mathrm{I})}=\frac{\mathbf{I}+2 a \sqrt{m}}{\mathrm{I}+2 a} e^{-2 a(\sqrt{m}-1)} .
$$

We have seen that for $b=o$ we get the Pareto distribution with $a=\alpha-\mathrm{I}$.

For Pareto the $m$ that maximizes $V-V_{\mathrm{I}}-V_{\mathrm{II}}$ had the property:

$$
\frac{\partial m}{\partial \alpha}=-\left(\frac{\mathrm{I}}{\alpha-2}\right)^{2}<0 .
$$

Now we have the same property for $a: \frac{\partial m}{\partial a}<0$.

\section{REFERENCES}

[I] Borch, K., "An Attempt to Determine the Optimum Amount of Stop Loss Reinsurance", XVIth International Congress of Actuaries, Brussels, r960.

[2] KaHN, P. M., "Some Remarks on a Recent Paper by Borch", ASTINColloquium, I96I, ASTIN-Bulletin, Vol. I, Part V, r96r.

[3] Verbeek, H., "On Optimal Reinsurance", ASTIN-Bulletin, Vol. IV, Part I, 1966.

[4] Benktander, G., "Schadenverteilung nach Grösse in der Nichtlebenversicherung", Mitteilungen der Vereinigung schweizerischer Versicherungsmathematiker, Band 70 , Heft 2. 